\title{
Telemedicina: aprovechando las tecnologías para favorecer el impacto social
}

\author{
Guillermo Rodolfo Friedrich $^{1}$, Jorge Raúl Ardenghi ${ }^{2}$ \\ ${ }^{1}$ Depto. Electrónica, Universidad Tecnológica Nacional, Facultad Regional Bahía Blanca, \\ 8000, Bahía Blanca Argentina \\ gfried@frbb.utn.edu.ar \\ ${ }^{1}$ Depto. Cs. e Ing. de la Computación, Universidad Nacional del Sur, 8000, Bahía Blanca, Argentina. \\ Laboratorio de Investigación en Sistemas Distribuidos (LiSiDi), miembro del IICyTI \\ (Instituto de Investigación en Ciencia y Tecnología Informática) \\ jra@cs.uns.edu.ar
}

\begin{abstract}
Resumen
En general la palabra telemedicina se asocia con cuestiones muy complejas y sofisticadas, como por ejemplo la realización de una cirugía a distancia, en la que están involucrados sistemas de comunicación, visualización y robótica basados en tecnologías de punta y de costos muy elevados. Si bien este tipo de aplicaciones es una de las más notorias dentro del campo de la telemedicina, tiene por ahora un costo muy elevado y un impacto social muy bajo. Si se plantea como un objetivo de la telemedicina acercar la atención médica a poblaciones alejadas de los centros urbanos, y que por lo general cuentan con recursos económicos limitados, el punto de vista es otro. En lugar de buscar el desarrollo de nuevas tecnologías de punta se debe tratar de aprovechar y potenciar las tecnologías existentes. Como la base de cualquier sistema de telemedicina es la infraestructura de comunicaciones, en el presente trabajo se analizan las posibilidades de algunas tecnologías de comunicación e incluso la complementación entre ellas.
\end{abstract}

Palabras clave: Telemedicina, Barrera Digital, GPRS, Enlace Inalámbrico.

\begin{abstract}
In general the "telemedicine” is associated with very complex and sophisticated tasks, like remote surgery, in which systems of communication, visualization and robotics are involved, based on advanced and expensive technologies. Although this type of application is well-known within the field of the telemedicine, so far is expensive and has a very low social impact. If one sets out as an objective of the telemedicine to approach the medical assistance to populations located far from the urban centers, and those people has limited economic resources, the point of view is another one. Instead of developping new technologies the goal is to exploit and power up the existing and deployed ones. As the base of any telemedicine system is the communications infrastructure, in this paper some communication technologies are analyzed and so are their complementation.
\end{abstract}

Palabras clave: Telemedicine, Digital Barrier, GPRS, Wireless.

\section{Introducción}

La telemedicina es una disciplina en la que convergen distintas ramas de la tecnología (comunicaciones, informática, robótica, etc.) y de la medicina (cirugía, clínica médica, etc.). Al hablar de telemedicina a muchos se le vendrá a la mente una operación quirúrgica realizada con los cirujanos en un continente y el paciente en otro. Esto involucra el uso de tecnología de punta en comunicaciones, procesamiento de imágenes, robótica, etc. El objetivo de semejante despliegue de recursos es acercar la medicina de alta complejidad a pacientes de cualquier lugar del mundo, evitando el traslado del paciente o de los especialistas. Sin embargo, en la actualidad estas técnicas están en etapa experimental y no son de aplicación corriente debido, entre otros factores, a sus elevados costos. Quien eventualmente podría acceder a estos servicios es una mínima proporción de la población, la misma que en general dispone de los recursos para viajar y hacerse atender incluso en otro continente.

$\mathrm{Si}$ se pretende que la telemedicina sirva para acercar la asistencia médica a regiones alejadas de los centros urbanos, debe tenerse en cuenta que en general 
esas poblaciones rurales tienen bajo nivel económico y no cuentan con tecnologías de punta para las comunicaciones [5]. En muchas de esas poblaciones por lo general no hay médico, sino que con suerte algún enfermero/a. En estos casos, la telemedicina puede tener una enorme importancia social, ya que sin pretender reemplazar la relación persona a persona entre médico y paciente, puede dar solución a una gran cantidad de situaciones: diagnósticos, consultas a especialistas, seguimiento de pacientes en tratamiento, etc.

En el presente trabajo no se trata del desarrollo de nuevas tecnologías de punta, sino de potenciar las tecnologías de comunicación existentes, con la finalidad de servir de infraestructura a sistemas de telemedicina que acerquen la atención médica primaria a los sectores de la sociedad que más lo necesitan.

\section{Metodología}

La base sobre la que se apoya cualquier sistema de telemedicina es la infraestructura de comunicación. Los requerimientos para el sistema de comunicación están impuestos por el tipo y caudal de información que se debe transferir. Una primer clasificación de la información a utilizar en un sistema de telemedicina podría ser la siguiente:

- Audio

- Video

- Señales

- Imágenes

- Archivos

En principio se puede considerar que el audio y el video es a los fines de la comunicación entre médico y paciente o entre el médico y el auxiliar que asiste al paciente. Las señales pueden provenir de distintos tipos de sensores, cuya finalidad es permitirle al médico conocer el estado de distintos parámetros y signos vitales del paciente, entre otros: frecuencia cardíaca, temperatura, presión, peso, estatura, etc. Esta lista se puede extender en la medida que se cuente con otros equipos de medición. Las imágenes, a diferencia del video, son estáticas pero con la definición necesaria para ayudar al diagnóstico o evaluación por parte del médico. Pensando en la atención primaria no es necesario remitirse a una imagen obtenida por resonancia magnética. Podría tratarse de cosas sencillas como una fotografía digital que ayude a evaluar la gravedad de una herida o quemadura, y posibilite ordenar el tratamiento adecuado y/o observar la evolución de un tratamiento. En caso de contarse en el lugar con un equipo de rayos $\mathrm{X}$, se podría digitalizar $\mathrm{y}$ transmitir la imagen de una radiografía.

En la actualidad el medio preferido para el transporte de información de los tipos más diversos es la Internet. Si en dos lugares del planeta se dispone de una conexión a Internet va a ser posible la comunicación entre ellos. Teniendo en cuenta las consideraciones anteriores, podríamos pensar que si ambos extremos (médico y paciente remoto) tuvieran un acceso de banda ancha a Internet, como se ve en la Fig. 1, estaría resuelta una gran parte del problema.

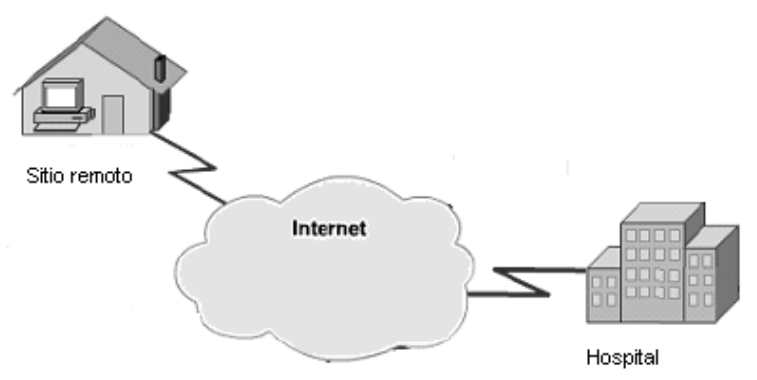

Figura 1. Conexión entre el médico en el hospital y el paciente remoto a través de Internet, mediante una conexión de banda ancha en cada extremo.

Es difícil que un hospital ubicado en un centro urbano no pueda tener acceso de banda ancha a Internet, sin embargo en extremo del paciente es donde se presentan los problemas. Se podría decir que si en el sitio remoto es posible tener una conexión adecuada a Internet, será posible el acceso a la telemedicina. La calidad y capacidad del acceso a Internet determinará las prestaciones con que se podrá contar. En algunas zonas rurales las alternativas de comunicación pueden ser:

- Telefonía básica

- Telefonía celular

La telefonía básica permite el acceso a Internet mediante un módem, con un ancho de banda limitado (entre 19.2 y 56 kbps) y un costo que estará en función de la duración de la comunicación y la distancia al ISP (Proveedor de Servicios de Internet). En caso de que la misma empresa de telefonía sea el ISP (por ejemplo: Telefónica de Argentina y Advance) el costo de comunicación podrá ser menor.

En caso de tener acceso a telefonía celular GSM, otra posibilidad es usar el servicio GPRS (General Packet Radio Service) [1] para el accceso a Internet. Esta es una alternativa muy interesante, debido a que es posible obtener un mayor ancho de banda y facilidad de despliegue que con la telefonía fija. Por otra parte, la tarifación en GPRS por lo general se efectúa en base a la cantidad de datos transferidos sin tener en cuenta el tiempo de conexión. Esto permite implementar un servicio del tipo siempre-conectado sin incurrir en mayores costos.

Algunos dispositivos que se pueden utilizar para conectar una computadora a Internet son:

- Teléfono celular con interfaz Bluetooth, IR o RS232.

- Router GPRS

- Modem GPRS

La figura 2 muestra como establecer una comunicación basada en TCP/IP, mediante un router GPRS 
[3]. Cabe señalar que las empresas de telefonía celular disponen de una pasarela entre su red GPRS e Internet.

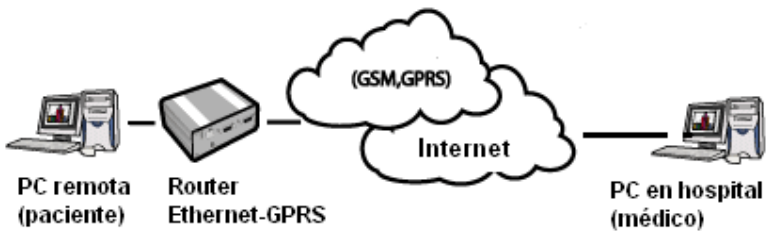

Figura 2. Conexión entre médico y paciente remoto mediante GSM/GPRS e Internet, usando un router GPRS.

A modo de ejemplo, el modem GSM/GPRS $\mu$ WEBox de Comtech [3] implementa, entre otras, las siguientes funciones: NAT usando la dirección IP dinámica obtenida en la red GPRS, servidor DHCP, maneja tráfico IP, TCP y UDP, capacidad hasta 10 sockets TCP simultáneos. La interfaz GPRS usa 4+1 canales, lo que permite esperar unos 40 48 kbps de bajada y 11 12 kbps de subida.

Sin embargo, es frecuente encontrar pequeñas poblaciones rurales que no disponen de telefonía fija y que además están separadas varios kilómetros de alguna zona con cobertura GSM. En estos casos es necesario aplicar alguna variante a la propuesta anterior, como puede ser la implementación de un enlace inalámbrico con tecnología Wi-Fi. Este caso se muestra en la figura 3 .

Mediante equipos de costo moderado, un par de Puntos de Acceso operando en la modalidad de puente y un par de antenas parabólicas, es posible cubrir una distancia de $30 \mathrm{~km}$ con un enlace de entre 1 y $11 \mathrm{Mbps}$ (si no se requiere más de $1 \mathrm{Mbps}$ se podría fijar esta velocidad, a fin de aumentar la robustez del vínculo). Este vínculo permitiría comunicar el equipo en el extremo del paciente con el router GPRS.

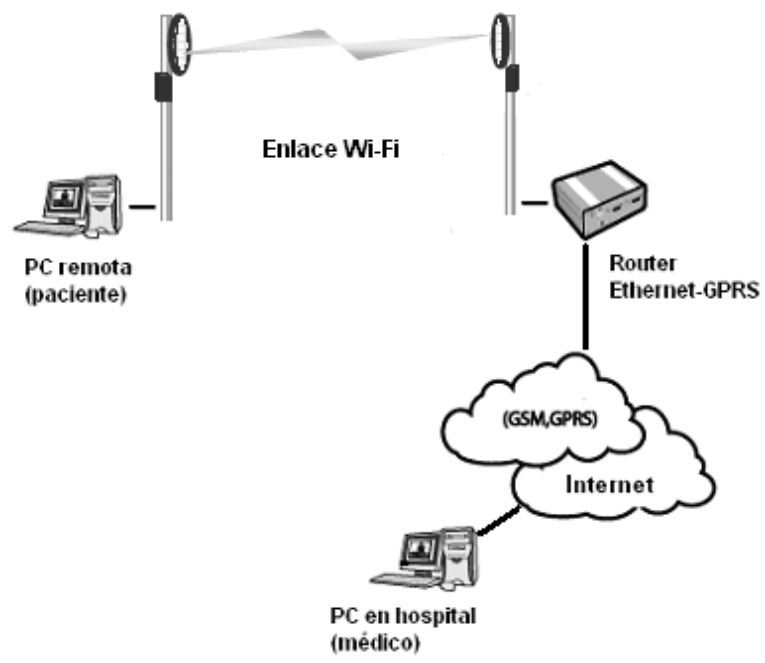

Figura 3. Conexión del extremo remoto (paciente) al router GPRS mediante un enlace Wi-Fi. Una solución posible para pequeñas poblaciones alejadas algunos km de una zona con cobertura GSM/GPRS.

Es posible implementar un enlace de este tipo utilizando equipos Wi-Fi de costo moderado, inclusive algunos que han sido diseñado para uso en interiores (agregándole alguna protección contra la intemperie). A modo de ejemplo se puede citar una experiencia realizada mediante un par de Puntos de Acceso Senao NL-2611CB3 PLUS, de $23 \mathrm{dBm}$ de potencia [7] y antenas grilladas de $27 \mathrm{dBi}$, mediante los que se implementó un enlace de $29 \mathrm{~km}$ a $1 \mathrm{Mbps}$ (a pesar de poder funcionar a mayor velocidad se fijó a 1 Mbps para favorecer la robustez).

\subsection{Ensayos sobre la red GPRS}

En un trabajo de Chakravorty y Pratt [2], en el que se evalúa la performance de la red GPRS con vistas a su utilización para el acceso a Internet, se pueden ver algunos resultados de interés. Algunos resultados importantes son la tasa de transferencia en subida y bajada, y el retardo de ida y vuelta (RTT).

Por otra parte en otro trabajo de los autores [4] se evaluaron las prestaciones de la red GPRS para aplicaciones de monitoreo remoto, las que son afines a ciertas tareas de telemedicina.

En las experiencias realizadas por ambos grupos se utilizaron modems GPRS, de tipo 3+1 (tres canales GSM para la bajada y uno para la subida). El modelo utilizado por los autores en el trabajo citado es un Motorola G20 [6]. Estos han sido diseñados para el acceso típico de un cliente a la Internet, en que el mayor caudal de tráfico viaja hacia el cliente. El router GPRS mencionado más arriba también ha sido diseñado con el mismo criterio (4+1). Esto es contrario a las necesidades de un sistema de telemedicina, debido a que la mayor producción de información estaría en el extremo remoto. Es de esperar que en el futuro se pueda contar con módems y routers GPRS con capacidades simétricas.

\section{$3 \quad$ Resultados}

Dentro de los distintos ensayos que se describen en [2], la medición de la tasa de transferencia bajo diferentes condiciones de señal da una idea básica de las posibilidades de GPRS para el tráfico de datos. Utilizando un módem de tipo 3+1 los resultados obtenidos son:

- Tráfico de bajada (3 canales):

- Con buenas condiciones de señal: 33 kbps.

- Con pobres condiciones de señal: 11 kbps.

- Tráfico de subida (1 canal):

- Con buenas condiciones de señal: 12 kbps.

- Con pobres condiciones de señal: 4 kbps. 
El nivel de señal es un factor cuya variación se hace sentir especialmente es equipos sujetos a movilidad. Sin embargo, como para el objetivo de este trabajo no interesa la movilidad que brinda esta red, sino su facilidad para el despliegue de sitios remotos, se puede considerar que será posible disponer los equipos de tal modo de trabajen con buena señal. Por lo tanto es de esperar una tasa de transferencia de aproximadamente $33 \mathrm{kbps}$ de bajada y $12 \mathrm{kbps}$ de subida. Estos valores son bastante escasos para llevar adelante una sesión con audio y video en tiempo real. Sin embargo se pueden mencionar algunos posibles ejemplos de uso:

- enviar una imagen que facilite el diagnóstico por parte del médico.

- enviar un archivo conteniendo el resultado de un exámen (por ejemplo, un electrocardiograma).

Asimismo, este ancho de banda puede ser suficiente para controlar en forma remota algún instrumental y para el monitoreo en tiempo real de algunas variables de los pacientes (por ej.: temperatura, presión sanguínea, etc.). Para este tipo de aplicaciones el tiempo de respuesta y la confiabilidad de la red pueden ser más importantes que el ancho de banda.

Uno de los ensayos realizados en [4] consistió en determinar el tiempo de retardo de ida y vuelta entre un equipo remoto conectado mediante un modem GSM/GPRS y un servidor en Internet. El cliente envía un datagrama UDP y el servidor lo devuelve a fin de que el cliente mida el tiempo transcurrido. En la figura 4 se pueden apreciar los resultados obtenidos. En condiciones de poca carga la red, el RTT promedio ronda los 1350 mseg, mientras que cuando la red está más cargada, el RTT promedio se sitúa alrededor de los 2 seg. Es muy raro percibir un RTT mayor a los 3 seg, por lo que este tiempo puede considerarse como el máximo para esperar una respuesta.

Distribución porcentual del RTT (en mseg.)

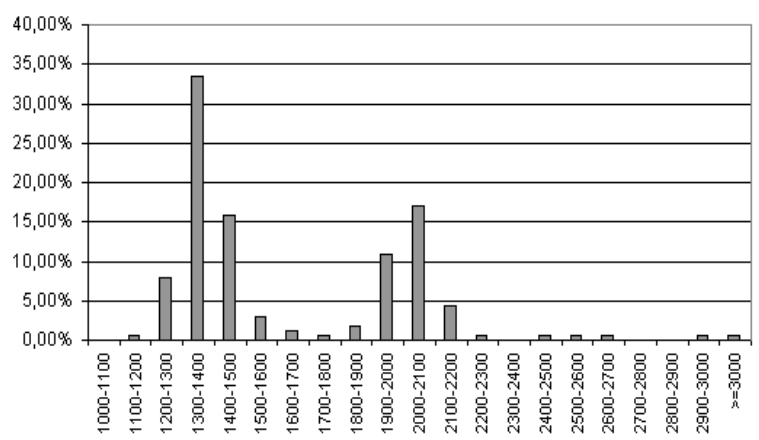

Figura 4. Distribución porcentual del RTT medido entre un cliente GPRS y un servidor en Internet.

Otro ensayo relacionado con el anterior consistió en enviar una secuencia de pings desde un host en Internet hasta el equipo conectado a la red GPRS, a fin de evaluar la variación en el RTT dentro de una secuencia de paquetes. Se puede observar que el primer ping tiene un retardo mucho mayor que para el resto. Asimismo, si en una secuencia de pings se pierde una respuesta, la próxima respuesta recibida sufre una demora mayor y las siguientes vuelven a valores menores. A continuación se muestra un fragmento de los tiempos obtenidos en una secuencia de pings:

$\begin{array}{ll} & \ldots . . \\ & 665 \mathrm{~ms} \\ & 681 \mathrm{~ms} \\ & 683 \mathrm{~ms} \\ & \text { Tiempo de espera agotado } \\ \rightarrow \quad & 1799 \mathrm{~ms} \\ & \text { Tiempo de espera agotado } \\ \rightarrow \quad & 1345 \mathrm{~ms} \\ & 693 \mathrm{~ms} \\ & \text { Tiempo de espera agotado } \\ & 1274 \mathrm{~ms} \\ & 702 \mathrm{~ms} \\ & 803 \mathrm{~ms} \\ & . . . .\end{array}$

Esta notable diferencia entre el primer paquete y los sucesivos se debe a que dentro de la red GPRS la información de enrutamiento permanece en caché por un tiempo muy breve (en el orden del segundo). La influencia que puede tener esta dispersión del RTT va a ser mayor para aquellas aplicaciones que trabajan en la modalidad de comando/respuesta. Por ejemplo: desde el sitio central se envía el comando para que un instrumento realice una tarea, el instrumento la realiza y envía la respuesta. Cabría esperar que la latencia para que el comando llegue a destino va a ser alta; asimismo, si el proceso que se debe ejecutar en respuesta al comando recibido tiene una duración del orden del segundo o más, cabe esperar que la respuesta también tenga una latencia alta. Es decir, que el RTT total podrá estar entre 2 y 4 seg sumados al tiempo de procesamiento del comando. Este retardo es importante de ser tenido en cuenta, debido a que afecta a la percepción de la persona que está operando algún instrumento en forma remota.

En otra serie de ensayos efectuados por medio de traceroute se pudo determinar que la mayor parte del mismo (80 90 \%), se produce al atravesar la red GSM. Cabe aclarar que el resto del trayecto entre la pasarela GPRS/Internet y el servidor o host conectado a Internet a través de un ISP, correspondió a enlaces terrestres dentro del mismo pais (Argentina).

\section{Discusión y Conclusiones}

Al hablar de telemedicina es habitual asociarla con el desarrollo de tecnologías de punta que permitan realizar a distancia tareas médicas de alta complejidad, como por ejemplo una cirugía. Sin embargo, éste no es el único punto de vista. En el presente trabajo se plantea como aprovechar la tecnología existente en el campo de las tecnologías de la información y las comunicaciones para acercar la posibilidad de atención 
médica a poblaciones alejadas de los centros urbanos, que por otra parte cuentan con pocos recursos.

Como la columna vertebral de un sistema de telemedicina es la infraestructura de comunicaciones, y teniendo en cuenta que en la actualidad la Internet es el medio de comunicación por excelencia, se analizaron algunas alternativas que posibiliten, de manera económica, el acceso a Internet a poblaciones alejadas de los centros urbanos. Se considera en especial el aprovechamiento de la red de telefonía celular GSM, a través del servicio GPRS, debido a su fácil integración con los protocolos TCP/IP y el acceso a Internet. Asimismo se considera su combinación con la tecnología Wi-Fi para implementar enlaces de hasta 30 $\mathrm{km}$, para permitir la llegada a una zona con cobertura GSM.

Si bien las propuestas de este trabajo pueden no ser una solución para poblaciones muy alejadas de los centros urbanos, si lo son para una gran cantidad de poblaciones separadas algunas decenas de kilómetros de algún punto con posibilidad de conexión a la red GSM o bien directamente a la Internet.

Una deficiencia de los modems y routers GPRS actuales es que su capacidad de tráfico es asimétrica $(3+1$ o 4+1), lo que resulta desfavorable para un sistema de telemedicina. Es de esperar que en el futuro estos equipos permitan el tráfico con capacidades simétricas.

En una próxima etapa se pretende realizar algunos ensayos de campo, a fin de evaluar las prestaciones que puede brindar un sistema de este tipo en condiciones reales y determinar los aspectos a mejorar.

\section{Referencias}

[1] BATES R. J. GPRS: General Packet Radio Service, McGraw-Hill.

[2] CHAKRAVORTY R. and PRATT I. Performance Issues with General Packet Radio Service, Journal of Communications and Networks (JCN), Special Issue on Evolving from 3G deployment to $4 \mathrm{G}$ definition, Vol. 4, No. 2, Dec. 2002.

[3] COMTECH. $\mu$ WEBox eRouter GSM / GPRS routers, disponible en formato pdf en: http://www.comtechm2m.com/brochure/eRouterfa mily.pdf

[4] FRIEDRICH G. R. y ARDENGHI J. R. Evaluación de las prestaciones de la red GPRS para su uso en aplicaciones de monitoreo remoto. Enviado al
CACIC (Congreso Argentino de Ciencias de la Computación) a realizarse en Octubre de 2006 en Potrero de los Funes, San Luis, Argentina.

[5] KMETEUK FILHO, O. \& RICHTER ANACLETO, C. Telemedicina: possibilidades versus realidade. Revista Eletrônica de Sistemas de Informaçâo (RESI), $5^{\circ}$ Ed., Year IV, Vol. IV, $\mathrm{N}^{\circ} 1$, May 2005 (http://www.inf.ufsc.br/resi).

[6] MOTOROLA. G20 AT Commands Developer's Guide Motorola, disponible en formato pdf en: http://www.motorola.com/mot/doc/0/970_MotDoc. zip

[7] SENAO. High Power (23dbm) Multi-Client Bridge / Access Point, NL-2611CB3 PLUS (Deluxe). Información disponible en: http://www.senao.com/ english/product/Product_wireless01_all.asp

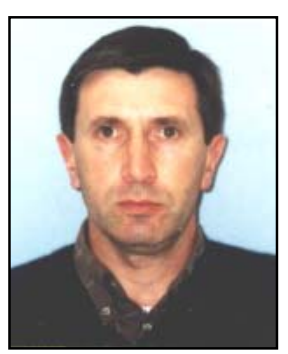

Guillermo Rodolfo Friedrich es Profesor Asociado en la Universidad Tecnológica Nacional, Facultad Regional Bahía Blanca, Argentina. Obtuvo sus títulos de Magíster en Cs. de la Computación en 2001 y de Ingeniero Electricista en 1986, en la Universidad Nacional del Sur, Argentina.

Integrante del LiSiDi (Laboratorio de Sistemas Distribuidos) de la UNS, y del Grupo de Estudios de Bioingeniería (UTN-FRBB). Sus áreas de interés son Redes y Arquitecturas para procesamiento digital de señales.

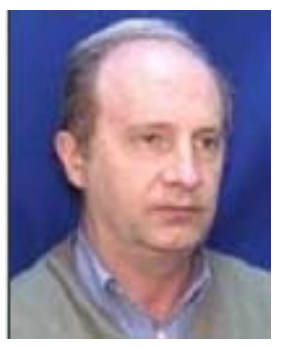

Jorge Raúl Ardenghi es Profesor Titular en la Universidad Nacional del Sur (UNS), en Bahía Blanca, Argentina, y Director del Laboratorio de Sistemas Distribuidos (LiSiDi) en la misma Universidad. Obtuvo su título de Magíster en Ing. Eléctrica en la Universidad de Chile (1973) y de Ingeniero Electricista en la UNS (1970). Sus áreas de interés son Sistemas Distribuidos y Paralelos, Clusters y Grids, Computación de Alto Rendimiento. 